\title{
GROWTH CONDITIONS FOR ENTIRE FUNCTIONS WITH ONLY BOUNDED FATOU COMPONENTS
}

\author{
AIMO HINKKANEN AND JOSEPH MILES
}

\begin{abstract}
Let $f$ be a transcendental entire function of order $<$ $1 / 2$. We denote the maximum and minimum modulus of $f$ by $M(r, f)=\max \{|f(z)|:|z|=r\}$ and $m(r, f)=\min \{|f(z)|:|z|=$ $r$. We obtain a minimum modulus condition satisfied by many $f$ of order zero that implies all Fatou components are bounded. A special case of our result is that if

$$
\log \log M(r, f)=O\left(\log r /(\log \log r)^{K}\right)
$$

for some $K>1$, then there exist $\alpha>1$ and $C>0$ such that for all large $R$, there exists $r \in\left(R, R^{\alpha}\right]$ with

$$
\frac{\log m(r, f)}{\log M(R, f)} \geq \alpha\left(1-\frac{C}{(\log \log R)^{K}}\right),
$$

and this in turn implies boundedness of all Fatou components.

The condition on $m(r, f)$ is a refined form of a minimum modulus conjecture formulated by the first author. We also show that there are some functions of order zero, and there are functions of any positive order, for which even refined forms of the minimum modulus conjecture fail. Our results and counterexamples indicate rather precisely the limits of the method of using the minimum modulus to rule out the existence of unbounded Fatou components.
\end{abstract}

\section{INTRODUCTION}

Let $f$ be a transcendental entire function. In 1981, I.N. Baker [2] proved that if the growth of the maximum modulus of $f$ does not exceed a certain rate, then all the components of the Fatou set of $f$ are bounded. He asked what would be the largest rate of growth that guarantees this conclusion, and showed that the best one can hope for is that the growth of $f$ does not exceed order $1 / 2$, minimal type.

We shall use the usual definitions of complex dynamics (see, e.g., [4], [5], [10], [14]). We denote the iterates of $f$ by $f^{1}=f$, and $f^{n}=f \circ f^{n-1}$ for $n \geq 2$. We say that $z \in \mathbb{C}$ lies in the Fatou set $\mathcal{F}(f)$ of $f$ if there

2000 Mathematics Subject Classification. Primary: 30D05; Secondary: 37F50. This material is based upon work supported by the National Science Foundation under Grant No. 0457291. 
exists a neighborhood $U$ of $z$ such that the family $\left\{f^{n} \mid U: n \geq 1\right\}$ of the restrictions of the iterates of $f$ to $U$ is a normal family. The Julia set $\mathcal{J}(f)$ of $f$ is $\mathcal{J}(f)=\mathbb{C} \backslash \mathcal{F}(f)$. The definition shows that the set $\mathcal{F}(f)$ is open. The set $\mathcal{J}(f)$ is non-empty and perfect, and is equal to $\mathbb{C}$ or is a nowhere dense subset of $\mathbb{C}$.

We write $M(r, f)=\max \{|f(z)|:|z|=r\}$ for the maximum modulus of $f$ and $m(r, f)=\min \{|f(z)|:|z|=r\}$ for the minimum modulus of $f$. The notation $m(r, f)$ is also the standard notation for the proximity function of $f$ in the Nevanlinna theory, and we will explicitly point out the one time where the notation $m(r, f)$ is used in that meaning. The order $\rho(f)$ and lower order $\lambda(f)$ of $f$ are defined by

$$
\rho(f)=\limsup _{r \rightarrow \infty} \frac{\log \log M(r, f)}{\log r}, \quad \lambda(f)=\liminf _{r \rightarrow \infty} \frac{\log \log M(r, f)}{\log r} .
$$

If $0<\rho(f)=\rho<+\infty$, we define the type of $f$ by

$$
\tau(f)=\limsup _{r \rightarrow \infty} \frac{\log M(r, f)}{r^{\rho}} .
$$

If $\tau(f)=0$, we say that $f$ is of minimal type. If $0<\tau(f)<+\infty$, we say that $f$ is of mean type. If $\tau(f)=+\infty$, we say that $f$ is of maximal type.

I.N. Baker [2] asked in 1981 whether every component of $\mathcal{F}(f)$ is bounded if the growth of $f$ is sufficiently small. The function

$$
f(z)=z^{-1 / 2} \sin \sqrt{z}+z+a
$$

is of order $1 / 2$, mean type, and if $a$ is a sufficiently large positive number, then $\mathcal{F}(f)$ has an unbounded component $D$ containing a segment $\left[x_{0}, \infty\right)$ of the positive real axis, such that $f^{n}(z) \rightarrow \infty$ as $n \rightarrow \infty$, locally uniformly in $D$. Baker noted ([2], p. 484) that it is possible to have a function of order $1 / 2$ and of arbitrarily small type with the same properties. Thus, while one might hope to prove that all components of $\mathcal{F}(f)$ are bounded provided that the growth of $f$ does not exceed order $1 / 2$, minimal type, one cannot do better.

For further background, we refer to the survey paper [8] of the first author. Here we only briefly mention the following results that have been previously obtained on this problem.

That all components of $\mathcal{F}(f)$ are bounded for a transcendental entire function $f$ was proved by Baker [2] under the assumption that $\log M(r, f)=O\left((\log r)^{p}\right)$ where $1<p<3$, and by Stallard [13] when

$$
\log \log M(r, f)=O\left(\frac{(\log r)^{1 / 2}}{(\log \log r)^{\varepsilon}}\right)
$$


for some $\varepsilon>0$. The latter is the best known condition so far based on growth alone.

When the regularity of growth of $M(r, f)$ is also taken into account, the following conditions are known to imply the boundedness of all components of $\mathcal{F}(f)$, where we assume throughout that the order of $f$ is $<1 / 2$. Stallard [13] proved that this is the case if there exists a real number $c \in[1, \infty)$ such that

$$
\lim _{r \rightarrow \infty} \frac{\log M(2 r, f)}{\log M(r, f)}=c
$$

Anderson and the first author [1] proved that all components of $\mathcal{F}(f)$ are bounded if there exists a positive constant $c$ such that for all sufficiently large $x$, the increasing convex function $\varphi(x)=\log M\left(e^{x}, f\right)$ satisfies $\varphi^{\prime}(x) / \varphi(x) \geq(1+c) / x$. This is true, in particular, if $f$ is of positive lower order ([8], Section 8).

Stronger results have been proved for particular types of components of $\mathcal{F}(f)$. Let $U$ be a periodic or preperiodic component of $\mathcal{F}(f)$ other than a Baker domain or a preimage of a Baker domain. Thus there is a positive integer $n$ such that the component $V$ of $\mathcal{F}(f)$ containing $f^{n}(U)$ belongs to an attracting, superattracting, or parabolic cycle of components of $\mathcal{F}(f)$, or to a cycle of Siegel disks. Baker [2] proved that if the growth of $f$ is at most order $1 / 2$, minimal type, then $U$ is bounded. Zheng [15] extended this result to the case when $V$ belongs to a cycle of Baker domains so that $\lim _{m \rightarrow \infty} f^{m}(z) \rightarrow \infty$ for $z \in V$ and $f^{q}(V) \subset V$ for some positive integer $q$ (Stallard [12] proved this for functions of order $<1 / 2)$.

The only components of $\mathcal{F}(f)$ not covered by these results are the wandering domains $U$, characterized by the property that for all distinct positive integers $m$ and $n$, we have $f^{m}(U) \cap f^{n}(U)=\emptyset$. If for an arbitrary transcendental entire function $f$, such a component $U$ is multiply connected, then by a result of Baker [3], all components of $\mathcal{F}(f)$ are bounded. Thus we may assume that all wandering domains of $f$ are simply connected.

The problem can thus be formulated as follows. Let $f$ be a transcendental entire function whose growth is at most order $1 / 2$, minimal type. Suppose that $f$ has at least one wandering domain and that all wandering domains of $f$ are simply connected. What else, if anything, needs to be assumed of $f$ to prove that all of its wandering domains are bounded (all other components of $\mathcal{F}(f)$ necessarily being bounded) ?

There could be many ways of approaching this problem. In this paper we consider this problem from the point of view of minimum 
modulus estimates for $f$. Our starting point is the following result of the first author [7].

Theorem 1.1. Let $f$ be a transcendental entire function of order $<$ $1 / 2$. Suppose that there exist positive numbers $R_{0}, L, \delta$, and $C$ with $R_{0}>e, M\left(R_{0}, f\right)>e, L>1$, and $0<\delta \leq 1$ such that for every $R>R_{0}$ there exists $r \in\left(R, R^{L}\right]$ with

$$
\frac{\log m(r, f)}{\log M(R, f)} \geq L\left(1-\frac{C}{(\log R)^{\delta}}\right) .
$$

Then all the components of the Fatou set of $f$ are bounded.

The idea of the proof of Theorem 1.1 is that to get a contradiction, we assume that $f$ has an unbounded simply connected wandering domain $U$, and then show that $U$ has a compact subset $K$ whose images under the iterates of $f$ persist in having a large radial spread. This firstly rules out the possibility of any subsequence of the $f^{n}$ having a finite (necessarily constant) limit function in $U$, so that $\lim _{n \rightarrow \infty} f^{n}(z)=\infty$ locally uniformly for $z \in U$. On the other hand, since each application of $f$ will not increase the hyperbolic distance between the points of $K$, measured in the distinct domains containing $f^{n}(U)$, one can show that in the long run, spreads of the kind obtained are not possible, which then yields the desired contradiction.

More precisely, using dynamics and the hyperbolic geometry, we deduce that once $K$ is given, there is a constant $C>1$ depending on $K$ such that

$$
\frac{1}{C} \leq \frac{\left|f^{n}(z)\right|}{\left|f^{n}(w)\right|} \leq C
$$

whenever $n \geq 1$ and $z, w \in K$. This is a limitation on the ratio of the moduli of two points. The lower estimate for the spread that we obtain from arguments that have nothing to do with dynamics applies, instead, to the ratio of the logarithms of the moduli of two points. We deduce that if $K$ is properly chosen to begin with (which choice then determines $C$, so that $C$ may be large) then for each $n$ there are $z_{n}, w_{n} \in f^{n}(K)$ such that

$$
\frac{\log \left|f\left(z_{n}\right)\right|}{\log \left|f\left(w_{n}\right)\right|} \geq L_{0}>1
$$

for a fixed $L_{0}>1$. It is clear that even if $C$ may be large and $L_{0}$ may be very close to $1,(2)$ and (3) will be incompatible when $n$ is so large that $\left|f^{n}(z)\right|$ and $\left|f^{n}(w)\right|$ will be sufficiently large as determined by $C$ and $L_{0}$. This last situation will occur since $\lim _{n \rightarrow \infty} f^{n}(z)=\infty$ uniformly for $z \in K$. This is how a contradiction is produced under 
these assumptions: dynamics limits the radial spread while the fact that the minimum modulus is large sufficiently often forces the radial spread to remain large.

In [7] the first author suggested that perhaps the assumptions of Theorem 1.1 are valid for all transcendental entire functions $f$ of order $<1 / 2$, which would then imply that for all such functions all components of $\mathcal{F}(f)$ are bounded. In this paper we give counterexamples to show that these assumptions do not hold provided that the growth of $f$ is fast enough. There are even counterexamples of zero order. Thus, if the growth of $f$ is sufficiently rapid, it will be necessary to use totally different methods, or other methods in addition to those provided by Theorem 1.1 and its proof, if one hopes to prove that all components of $\mathcal{F}(f)$ are bounded.

However, we also prove that if the growth of $f$ is not too fast, then conditions that are close to the assumptions of Theorem 1.1 are satisfied, and as a result, all components of $\mathcal{F}(f)$ are bounded. Some technical modifications in the assumptions of Theorem 1.1 are necessary as Theorem 1.1 was clearly only a tentative result, its proof being based on choosing a particular convergent series and formulating the assumption accordingly. If one wishes to take this technique to its limit, one must consider an arbitrary convergent series with positive terms.

This provides a condition based on growth alone that is stronger than that obtained by Stallard [13] and that is sufficient to imply the boundedness of the components of $\mathcal{F}(f)$. Perhaps of greater interest is the fact that our results, taken together, illustrate the limits of this method of proof based on the minimum modulus alone. Thus further results should require a more careful study of factors other than the modulus of the function, such as, perhaps, the behavior of the argument of the function and the more precise structure, or shape, of the hypothetical unbounded simply connected wandering domains of $f$. We hope to be able to return to this latter subject in another paper.

\section{Results}

\subsection{Growth rate guaranteeing the boundedness of all Fatou} components. First we present our positive results to the effect that if a transcendental entire function does not grow too fast, then a modified form of the minimum modulus conjecture of the first author [7] is valid, and as a consequence, all components of the Fatou set of the function are bounded. This is achieved through the combination of the following three theorems. 
Theorem 2.1. Suppose that $f$ is a transcendental entire function of order 0 . For $r>e$, write

$$
\beta(r)=\sup \left\{\frac{\log ^{+} \log ^{+} M(t, f)}{\log t}: t \geq r\right\} .
$$

Suppose that $\gamma:(e,+\infty) \rightarrow(0,+\infty)$ is such that

$$
\begin{aligned}
& \text { (i) } \gamma(r) \rightarrow 0 \quad \text { as } r \rightarrow \infty, \quad \text { and } \\
& \text { (ii) } \frac{\gamma(r)}{\beta(r)} \rightarrow \infty \quad \text { as } r \rightarrow \infty \text {. }
\end{aligned}
$$

Then for each $\alpha>1$ and all large $R$, there exists $r \in\left(R, R^{\alpha}\right]$ such that

$$
\frac{\log m(r, f)}{\log M(R, f)} \geq \alpha(1-3 \gamma(R)) \text {. }
$$

Theorem 2.2. Let $f$ be a transcendental entire function of order $<$ $1 / 2$. Suppose that there exist $\alpha>1, R_{0}>1$, and a positive decreasing function $\gamma(r)$ such that for every $R>R_{0}$ there exists $r \in\left(R, R^{\alpha}\right]$ with

$$
\frac{\log m(r, f)}{\log M(R, f)} \geq \alpha(1-\gamma(R))
$$

and $\sum_{n} \gamma\left(e^{2^{n}}\right)<\infty$. Then all the components of the Fatou set of $f$ are bounded.

Theorem 2.3. Suppose that $f$ is a transcendental entire function of order 0 . Let $\beta(r)$ be defined as in Theorem 2.1. If

$$
\sum_{n} \beta\left(e^{2^{n}}\right)<\infty
$$

then $f$ has no unbounded Fatou component.

To satisfy the condition $\sum_{n} \beta\left(e^{2^{n}}\right)<\infty$ in Theorem 2.3 it suffices to have, for example,

$$
\beta(r) \leq \frac{1}{(\log \log r)(\log \log \log r) \cdots\left(\log _{j} r\right)^{K}}
$$

for all large $r$ and some $j \geq 2$, where $K>1, \log _{1} r=\log r$ and $\log _{j} r=\log \left(\log _{j-1} r\right)$.

\subsection{Growth rate beyond which the minimum modulus conjec-}

ture is not valid. Next we turn to a counterexample. Theorem 2.4 enables us to determine a growth rate for entire functions of order zero beyond which our method is not effective. 
Theorem 2.4. Suppose that $\beta(r)$ and $\gamma(r)$ are positive functions defined for $r>e^{3}$ such that

$$
\beta(r)>\frac{1}{(\log \log r)^{2}}
$$

for all $r>e^{3}, \beta(r)$ is decreasing, $\beta(r) \log r$ is increasing, and, as $r \rightarrow$ $\infty$, we have $\beta(r) \rightarrow 0, \gamma(r) \rightarrow 0$, and $\beta(r) / \gamma(r) \rightarrow \infty$. Suppose that $\alpha>1$. Then there exists an entire function $f$ such that for all large $r$, we have

$$
\sup \left\{\frac{\log ^{+} \log ^{+} M(t, f)}{\log t}: t \geq r\right\} \leq \beta(r),
$$

and also such that there exist arbitrarily large $R$ for which

$$
\frac{\log m(r, f)}{\log M(R, f)}<\alpha(1-\gamma(R)), \quad \text { for all } r \text { with } R<r \leq R^{\alpha} .
$$

The assumptions of Theorem 2.4 that $\beta(r)$ is decreasing while $\beta(r) \log r$ is increasing are natural since these properties hold if $\beta(r)$ is replaced by the left hand side of (8). In view of (7), $\beta(r) \log r \rightarrow \infty$ as $r \rightarrow \infty$.

Application. If the function $\beta$ is as in Theorem 2.4 and $\sum_{n} \beta\left(e^{2^{n}}\right)=$ $\infty$, Theorem 2.4 and its proof imply that there is no function $\gamma(r)$ to which Theorem 2.2 can be applied to show for all entire $f$ satisfying (8) that such $f$ have no unbounded Fatou components. For if $\sum_{n} \beta\left(e^{2^{n}}\right)=\infty$, then there exists $\gamma^{*}$ satisfying the assumptions of Theorem 2.4 in place of $\gamma$ such that $\sum_{n} \gamma^{*}\left(e^{2^{n}}\right)=\infty$. A cursory examination of the proof of Theorem 2.4 shows there is enough freedom in the construction that for all large $R$ there exists an entire function $f=f_{R}$ satisfying (8) for all large $r$ and (9). If $\gamma$ is a function to which Theorem 2.2 can be applied for all $f$ satisfying (8), we conclude from (6) and (9) that $\gamma(R)>\gamma^{*}(R)$ for all large $R$. Since $\sum_{n} \gamma\left(e^{2^{n}}\right)<\infty$, this is a contradiction, Our method of proof is thus effectively limited to functions satisfying the hypotheses of Theorem 2.3.

Remark. After preparing this paper, we learned that results closely related to ours have also been obtained by Rippon and Stallard; see [11].

\section{Proof of Theorem 2.1}

We employ the usual definitions and notation of the Nevanlinna theory as given, for example, in [6], except that, as noted, $m(r, f)$ denotes the minimum modulus of $f$ unless otherwise stated.

Let $f$ satisfy the assumptions of Theorem 2.1. We may find $a \in(0,1]$, $b \in \mathbb{C} \backslash\{0\}$ and a non-negative integer $k$ so that if $H(z)=f(a z) /\left(b z^{k}\right)$, 
then $H(0)=1$ and $H$ has no zeros in the unit disk. The functions $\beta(r)$ defined for $f$ and $H$ are asymptotic to one another; hence $H$ satisfies hypothesis (ii) of Theorem 2.1 as well. Therefore we assume for the time being that $f(0)=1$ and that $f$ has no zeros in the unit disk, and indicate later how to proceed in the general case.

We first note for all large $r$ that

$$
\gamma(r) \log r>\beta(r) \log r \geq \log ^{+} \log ^{+} M(r, f)>>1
$$

and for all $t>\sqrt{e}$ that

$$
n(t, 0, f) \log t \leq N\left(t^{2}, 0, f\right) \leq \log M\left(t^{2}, f\right) \leq \exp \left\{2 \beta\left(t^{2}\right) \log t\right\} .
$$

For large $R$, define $R_{0}=R_{0}(R)>R$ by

$$
\log R_{0}=\alpha(\log R)(1-\gamma(R)) .
$$

Denote the zeros of $f$ by $z_{n}=r_{n} e^{i \theta_{n}}$, with multiple zeros repeated according to their multiplicity. Write $f=F G$, where

$$
F(z)=\prod_{r_{n}<R_{0} / 10}\left(1-\frac{z}{z_{n}}\right)
$$

and

$$
G(z)=\prod_{r_{n} \geq R_{0} / 10}\left(1-\frac{z}{z_{n}}\right) .
$$

Suppose that $R_{0}+1<r<R^{\alpha}$. First consider a zero $z_{n}$ of $F$. Certainly

$$
\min \left\{\log \left|1-\frac{r e^{i \theta}}{z_{n}}\right|: 0 \leq \theta \leq 2 \pi\right\}=\log \left(\frac{r}{r_{n}}-1\right)
$$

and

$$
\max \left\{\log \left|1-\frac{R e^{i \theta}}{z_{n}}\right|: 0 \leq \theta \leq 2 \pi\right\}=\log \left(1+\frac{R}{r_{n}}\right) .
$$

For $1 \leq x \leq R_{0} / 10$, set

$$
h(x)=\frac{\log \left(\frac{r}{x}-1\right)}{\log \left(\frac{R}{x}+1\right)} .
$$

Claim: For $1<x<R_{0} / 10$, we have $h^{\prime}(x)>0$.

We now justify the claim. We have

$$
h^{\prime}(x)=\frac{R\left(R x+x^{2}\right)^{-1} \log \left(\frac{r}{x}-1\right)-r\left(r x-x^{2}\right)^{-1} \log \left(\frac{R}{x}+1\right)}{\left(\log \left(\frac{R}{x}+1\right)\right)^{2}},
$$

which has the same sign as

$$
\frac{R}{R+x} \log \left(\frac{r}{x}-1\right)-\frac{r}{r-x} \log \left(\frac{R}{x}+1\right)=I-I I,
$$


say. We analyze $I-I I$ on three different intervals:

Interval A: $10 R \leq x \leq R_{0} / 10$. For $x$ in interval $\mathrm{A}$ we have

$$
\frac{R}{R+x} \geq \frac{10 R}{11 x}
$$

and

$$
\frac{r}{x}>\frac{R_{0}}{x} \geq 10
$$

Hence

$$
I>\frac{10 R}{11 x} \log 9
$$

and

$$
\frac{r}{r-x} \leq \frac{r}{r-\frac{r}{10}}=\frac{10}{9} .
$$

Thus

$$
I I<\frac{10 R}{9 x}<\left(\frac{10}{11} \log 9\right) \frac{R}{x}<I .
$$

Hence $h^{\prime}(x)>0$.

Interval B: Since $\alpha>1$, we may choose $\omega$ such that

$$
\frac{23-\alpha}{22}<\omega<1
$$

Let interval $\mathrm{B}$ be $R^{\omega} \leq x<10 R$. We have

$$
\frac{R}{R+x} \geq \frac{R}{R+10 R}=\frac{1}{11}
$$

and

$$
\log \left(\frac{r}{x}-1\right)>\frac{1}{2} \log \frac{r}{x} \geq \frac{1}{2}(\log r-\log R-\log 10)>\frac{\eta-1}{2} \log R,
$$

for each $\eta<\alpha$ if $R>R^{*}(\eta)$.

Thus $I>\frac{\eta-1}{22} \log R$ for $\eta<\alpha, R>R^{*}(\eta)$. We have as well

$$
\frac{r}{r-x} \leq \frac{r}{r-10 R}=1+o(1), \quad R \rightarrow \infty .
$$

We have

$$
\begin{aligned}
\log \left(\frac{R}{x}+1\right) & =\log \frac{R}{x}+\log \left(1+\frac{x}{R}\right) \\
& \leq \log R-\omega \log R+\frac{x}{R} \\
& \leq(1-\omega) \log R+10
\end{aligned}
$$

Thus

$$
I I<(1+o(1))((1-\omega) \log R+10) .
$$


Since $1-\omega<\frac{\alpha-1}{22}$, there exists $\eta<\alpha$ such that for all large $R$

$$
I I<\frac{\eta-1}{22} \log R \text {. }
$$

Combining, we conclude for large $R$ that

$$
I-I I>0 \text {. }
$$

Thus $h^{\prime}(x)>0$ for $x$ in interval B.

Interval C: Suppose that $1 \leq x \leq R^{\omega}$. We have

$$
\begin{aligned}
\frac{I}{I I} & =\frac{R}{r}\left(\frac{r-x}{R+x}\right) \frac{\log \left(\frac{r}{x}-1\right)}{\log \left(\frac{R}{x}+1\right)} \\
& =\frac{1-\frac{x}{r}}{1+\frac{x}{R}}\left(1+\frac{\log \left(\frac{r}{x}-1\right)-\log \left(\frac{R}{x}+1\right)}{\log \left(\frac{R}{x}+1\right)}\right) \\
& \geq \frac{1-\frac{x}{r}}{1+\frac{x}{R}}\left(1+\frac{\log (r-x)-\log (R+x)}{\log (R+1)}\right) .
\end{aligned}
$$

Suppose that $1<\eta<\alpha$. For large $R$ we have $r>R^{\eta}$. Since $x<R$, and since we may assume that $2<R^{\eta-\omega}$, we have

$$
\log (r-x)>\eta \log R-\log 2
$$

and

$$
\log (R+x)<\log R+\log 2
$$

Thus

$$
\begin{aligned}
1+\frac{\log (r-x)-\log (R+x)}{\log (R+1)} & >1+\frac{(\eta-1) \log R-2 \log 2}{\log (R+1)} \\
& >\eta^{\prime} \text { for some } \eta^{\prime}>1, \text { if } R>R^{*}\left(\eta^{\prime}\right) .
\end{aligned}
$$

Since $x / r$ and $x / R$ are both $o(1)$ in interval $\mathrm{C}$, we see that $I / I I>1$. This shows that $h^{\prime}(x)=I-I I>0$ for $x$ in interval $\mathrm{C}$ when $R$ is large, and proves the claim.

For each zero $z_{n}=r_{n} e^{i \theta_{n}}$ of $F$ we have $1 \leq r_{n}<R_{0} / 10$, and by the claim and (10) we conclude for $R_{0}+1<r<R^{\alpha}$ that

$h\left(r_{n}\right) \geq h(1) \geq \frac{\log R_{0}}{\log (R+1)}=\frac{\alpha(\log R)(1-\gamma(R))}{\log (R+1)} \geq \alpha(1-2 \gamma(R))=\alpha_{R}^{\prime}$,

say, implying that

$$
\begin{aligned}
\log m(r, F) & \geq \sum_{r_{n}<R_{0} / 10} \log \left(\frac{r}{r_{n}}-1\right)=\sum_{r_{n}<R_{0} / 10} h\left(r_{n}\right) \log \left(\frac{R}{r_{n}}+1\right) \\
(12) & >\alpha_{R}^{\prime} \sum_{r_{n}<R_{0} / 10} \log \left(\frac{R}{r_{n}}+1\right)>\alpha_{R}^{\prime} \log M(R, F),
\end{aligned}
$$


for $R_{0}+1<r<R^{\alpha}$.

We now consider the factor $G(z)$. Set $P^{4}=R^{\alpha} / R_{0}$. Note that

$$
4 \log P=\alpha \log R-\log R_{0}=\alpha(\log R) \gamma(R)
$$

Thus

$$
\log P=\frac{\alpha(\log R) \gamma(R)}{4} .
$$

For $n=1,2,3, \ldots$, define $R_{n}=R_{0} P^{n}$, and note that $R_{4}=R^{\alpha}$. Set $I_{n}=\left[R_{n}, R_{n+1}\right)$ for $n \geq 0$. Note that

$$
\left[R_{0}, R^{\alpha}\right)=I_{0} \cup I_{1} \cup I_{2} \cup I_{3} .
$$

Case I: Suppose that $G$ has no zeros of modulus less than $R_{2}$. Then

$$
\log m\left(R_{1}, G\right) \geq \sum_{r_{n} \geq R_{2}} \log \left(1-\frac{R_{1}}{r_{n}}\right) .
$$

If $r_{n} \in I_{j}$ for $j \geq 2$, then

$$
\begin{aligned}
\log \left(1-\frac{R_{1}}{r_{n}}\right) & \geq \log \left(1-\frac{R_{1}}{R_{j}}\right)>\frac{-2 R_{1}}{R_{j}}=\frac{-2}{P^{j-1}} \\
& =-2 \exp (-(j-1) \log P) \\
& =-2 \exp \left\{\frac{-\alpha(j-1)(\log R) \gamma(R)}{4}\right\} .
\end{aligned}
$$

Let $n_{j}$ be the number of $z_{n}$ counted according to multiplicity with $\left|z_{n}\right| \in I_{j}$. We have from (11) that

$$
\begin{aligned}
n_{j} & <n\left(R_{j+1}, 0, G\right) \leq \exp \left\{2 \beta\left(R_{j+1}^{2}\right) \log R_{j+1}\right\} \\
& =\exp \left\{2 \beta\left(R_{j+1}^{2}\right)\left(\log R_{0}+(j+1) \log P\right)\right\} \\
& =\exp \left\{2 \beta\left(R_{j+1}^{2}\right)\left(\log R_{0}+\frac{(j+1) \alpha \gamma(R) \log R}{4}\right)\right\} .
\end{aligned}
$$

Thus, with

$$
X_{j}=2 \beta\left(R_{j+1}^{2}\right)\left(\log R_{0}+\frac{(j+1) \alpha \gamma(R) \log R}{4}\right)
$$

and

$$
Y_{j}=X_{j}-\frac{(j-1) \alpha \gamma(R) \log R}{4}
$$


we have

$$
\begin{aligned}
\log m\left(R_{1}, G\right) & \geq \sum_{j=2}^{\infty} \sum_{r_{n} \in I_{j}} \log \left(1-\frac{R}{r_{n}}\right) \\
& \geq-2 \sum_{j=2}^{\infty} n_{j} \exp \left\{-\frac{(j-1) \alpha \gamma(R) \log R}{4}\right\} \\
& \geq-2 \sum_{j=2}^{\infty} \exp \left\{Y_{j}\right\} \geq-2
\end{aligned}
$$

since this series is dominated by a rapidly converging geometric series with the first term and ratio both less than $\exp \{-(\alpha \gamma(R) \log R) / 2\}$. Recall that $\beta\left(R_{j+1}^{2}\right)$ is much smaller than $\gamma(R)$ for all $j \geq 2$, and, by (10), that $\gamma(R) \log R$ is large for large $R$.

Also, with

$$
Z_{j}=X_{j}+(1-\alpha) \log R+\alpha \gamma(R) \log R-j \log P
$$

we have

$$
\begin{aligned}
& \log M(R, G) \leq \sum_{j=2}^{\infty} \sum_{r_{n} \in I j} \log \left(1+\frac{R}{r_{n}}\right) \\
\leq & \sum_{j=2}^{\infty} n_{j} \frac{R}{R_{j}} \leq \sum_{j=2}^{\infty} \exp \left\{X_{j}\right\} \exp \left\{\log R-\log R_{j}\right\} \\
= & \sum_{j=2}^{\infty} \exp \left\{Z_{j}\right\}<2 \exp \left\{\left(\frac{1-\alpha}{2}\right) \log R\right\}=2\left(\frac{1}{R}\right)^{(\alpha-1) / 2},
\end{aligned}
$$

since the last series is dominated by a rapidly converging geometric series with first term less than $\exp \{((1-\alpha) / 2) \log R\}$ and ratio less than

$$
\exp \left\{-\frac{\alpha \gamma(R) \log R}{8}\right\} .
$$

Note that again we use the facts that $\beta\left(R_{j+1}^{2}\right)$ is much smaller than $\gamma(R)$ for $j \geq 2$ and that $\gamma(R) \log R$ is large for large $R$.

Combining, we get

$$
\begin{aligned}
\log m\left(R_{1}, f\right) & \geq \log m\left(R_{1}, F\right)+\log m\left(R_{1}, G\right) \\
& >\alpha_{R}^{\prime} \log M(R, F)-2 .
\end{aligned}
$$


Also,

$$
\begin{aligned}
\log M(R, f) & \leq \log M(R, F)+\log M(R, G) \\
& <\log M(R, F)+2\left(\frac{1}{R}\right)^{(\alpha-1) / 2} .
\end{aligned}
$$

Thus

$$
\begin{aligned}
\frac{\log m\left(R_{1}, f\right)}{\log M(R, f)} & \geq \frac{\alpha_{R}^{\prime} \log M(R, F)-2}{\log M(R, F)+2\left(\frac{1}{R}\right)^{(\alpha-1) / 2}} \\
& =\alpha_{R}^{\prime}-\frac{2 \alpha_{R}^{\prime}\left(\frac{1}{R}\right)^{(\alpha-1) / 2}+2}{\log M(R, F)+2\left(\frac{1}{R}\right)^{(\alpha-1) / 2}} \\
& >\alpha_{R}^{\prime}-\frac{o(1)}{\log R} \\
& >\alpha(1-3 \gamma(R)) \\
& =: \alpha_{R}^{\prime \prime} \quad \text { for large } R,
\end{aligned}
$$

where we have again used (10).

Case II: Suppose that $G$ has a zero in $\left\{z: R_{0} / 10 \leq|z|<R_{2}\right\}$. Clearly then there exists $t^{*} \in I_{2}=\left[R_{2}, R_{3}\right)$ such that $\log N\left(t^{*}, 0, G\right)>$ 0 .

Claim: For large $R$ if $t \geq R_{4}$, then

$$
\frac{\log N(t, 0, G)-\log N\left(t^{*}, 0, G\right)}{\log t-\log t^{*}}<\frac{1}{10} .
$$

We now justify the claim. To get a contradiction, suppose that the claim is false. Then there exists $t \geq R_{4}$ such that

$$
\log N(t, 0, G)-\log N\left(t^{*}, 0, G\right) \geq \frac{1}{10}\left(\log t-\log t^{*}\right)
$$


Now

$$
\begin{aligned}
\log t-\log t^{*} & =\frac{\log t-\log t^{*}}{\log t}(\log t) \\
& =\left(1-\frac{\log t^{*}}{\log t}\right) \log t \\
& >\left(1-\frac{\log R_{3}}{\log R_{4}}\right) \log t \\
& =\frac{\log \left(R_{4} / R_{3}\right)}{\log R_{4}} \log t \\
& =\frac{\log P}{\log R_{4}}(\log t) \\
& =\frac{\alpha \gamma(R)(\log R)(\log t)}{4 \log R_{4}} .
\end{aligned}
$$

Thus if there exists $t \geq R_{4}$ such that (14) is false, then

$$
\begin{aligned}
10 \log N(t, 0, G) & >10 \log N(t, 0, G)-10 \log N\left(t^{*}, 0, G\right) \\
& \geq \frac{\alpha \gamma(R) \log R}{4 \log R_{4}} \log t=\frac{\gamma(R)}{4} \log t,
\end{aligned}
$$

implying that

$$
\gamma(R) \log t<40 \log N(t, 0, G)<40 \beta(t) \log t,
$$

which is a contradiction for large $R$ since $t>R$. This establishes the claim.

It follows that there exists $t_{1} \in\left(R_{0} / 10, R^{\alpha}\right)$ such that

$$
\log N(t, 0, G) \leq \log \left(N\left(t_{1}, 0, G\right)\left(\frac{t}{t_{1}}\right)^{1 / 10}\right), \quad \text { for } \quad \frac{R_{0}}{10}<t<\infty .
$$

To see this, consider the graph of $\log N(t, 0, G)$ as a function of $\log t$, for $t \geq R_{0} / 10$, and the line $L$ on that graph of slope $1 / 10$ passing through $\left(\log t^{*}, \log N\left(t^{*}, 0, G\right)\right)$. The claim asserts that the graph of $\log N(t, 0, G)$ as a function of $\log t$ for $t \geq R^{\alpha}$ lies below $L$. Now consider parallel translates of $L$ (translation upward) until we obtain the highest translate $L^{\prime}$ of $L$ that intersects the graph; a point of intersection of the graph with this highest translate is a suitable point $\left(\log t_{1}, \log N\left(t_{1}, 0, G\right)\right)$. Any such $t_{1}$ lies in $\left(R_{0} / 10, R^{\alpha}\right)$, and we choose $t_{1}$ to be as large as possible.

If we write

$$
c_{m}(r, G)=\frac{1}{2 \pi} \int_{0}^{2 \pi} e^{-i m \theta} \log \left|G\left(r e^{i \theta}\right)\right| d \theta
$$


usual estimates (see [9]) give for all integers $m$

$$
\left|c_{m}\left(t_{1}, G\right)\right| \leq N\left(t_{1}, 0, G\right) \frac{\left(\frac{1}{10}\right)^{2}}{\left|\left(\frac{1}{10}\right)^{2}-m^{2}\right|}
$$

implying, after an application of Jensen's Theorem, that

$$
\log m\left(t_{1}, G\right)=\min \left\{\log \left|G\left(t_{1} e^{i \theta}\right)\right|: 0 \leq \theta \leq 2 \pi\right\}>0 .
$$

Claim: In fact $t_{1} \in\left(R_{0}+1, R^{\alpha}\right)$.

We now justify the claim. Note that

$$
\left.\frac{d(\log N(t, 0, G))}{d(\log t)}\right|_{t=t_{1}}=\frac{n\left(t_{1}, 0, G\right)}{N\left(t_{1}, 0, G\right)}=\frac{1}{10} .
$$

But if $R_{0} / 10<t_{1} \leq R_{0}+1$ then for all $r_{n}<t_{1}$ with $G\left(r_{n} e^{i \theta_{n}}\right)=0$ for some $\theta_{n}$, we have $\log \left(t_{1} / r_{n}\right)<\log 11$. It follows that

$$
N\left(t_{1}, 0, G\right)=\sum_{R_{0} / 10 \leq r_{n}<t_{1}} \log \frac{t_{1}}{r_{n}}<n\left(t_{1}, 0, G\right) \log 11
$$

Combining, we get

$$
\frac{1}{\log 11}<\frac{n\left(t_{1}, 0, G\right)}{N\left(t_{1}, 0, G\right)}=\frac{1}{10}
$$

which is a contradiction. This establishes the claim.

We have from (12)

$$
\begin{aligned}
\log m\left(t_{1}, f\right) & \geq \log m\left(t_{1}, F\right)+\log m\left(t_{1}, G\right) \\
& \geq \log m\left(t_{1}, F\right) \geq \alpha_{R}^{\prime} \log M(R, F)
\end{aligned}
$$

since $R_{0}+1<t_{1}<R^{\alpha}$.

By estimates very analogous to those leading to (13) (this time considering possible zeros of $G$ with modulus between $R_{0} / 10$ and $R_{2}$ as well as those of modulus greater than $R_{2}$ ), we again have

$$
\log M(R, G)<2\left(\frac{1}{R}\right)^{(\alpha-1) / 2}, \quad \text { for } \quad R>R^{*}, \quad \text { say. }
$$


Combining, we have

$$
\begin{aligned}
\frac{\log m\left(t_{1}, f\right)}{\log M(R, f)} & \geq \frac{\alpha_{R}^{\prime} \log M(R, F)}{\log M(R, F)+\log M(R, G)} \\
& \geq \frac{\alpha_{R}^{\prime} \log M(R, F)}{\log M(R, F)+\left(\frac{1}{R}\right)^{(\alpha-1) / 2}} \\
& =\alpha_{R}^{\prime}-\frac{\alpha_{R}^{\prime}\left(\frac{1}{R}\right)^{(\alpha-1) / 2}}{\log M(R, F)+\left(\frac{1}{R}\right)^{(\alpha-1) / 2}} \\
& =\alpha_{R}^{\prime}-\frac{o(1)}{\log R}>\alpha_{R}^{\prime \prime}, \quad \text { for } \quad R>R^{*} .
\end{aligned}
$$

Combined with the conclusion in Case I, this proves Theorem 2.1 when $f(0)=1$ and $f$ has no zeros in the unit disk.

Consider then the general case and define $H(z)=f(a z) /\left(b z^{k}\right)$, where $a \in(0,1], b \in \mathbb{C} \backslash\{0\}$ and $k$ is a non-negative integer so that $H(0)=1$ and $H$ has no zeros in the unit disk. The argument above proves Theorem 2.1 for $H$ instead of $f$, and we write $H=F G$ in what follows.

If we are in Case I, we recall that

$$
\begin{aligned}
& \log R_{1}=\log R_{0}+\log P=\alpha(\log R)(1-\gamma(R))+\alpha(\log R) \gamma(R) / 4 \\
& =\alpha(\log R)(1-(3 / 4) \gamma(R))
\end{aligned}
$$

and obtain for large $R$

$$
\begin{aligned}
& \log m\left(a R_{1}, f\right)=k \log R_{1}+\log m\left(R_{1}, H\right)+\log |b| \\
& \geq k \log R_{1}+\alpha_{R}^{\prime} \log M(R, F)-2+\log |b| \\
& \geq k \log R_{1}-2+\log |b|+\alpha_{R}^{\prime}(\log M(R, H)-\log M(R, G)) \\
& >k \log R_{1}-2+\log |b|+\alpha_{R}^{\prime}\left(\log M(R, H)-2\left(\frac{1}{R}\right)^{(\alpha-1) / 2}\right) \\
& \geq \alpha_{R}^{\prime} \log M(a R, f)+k\left(\log R_{1}-\alpha_{R}^{\prime} \log R\right)+O(1) \\
& \geq \alpha_{R}^{\prime} \log M(a R, f)+k \alpha(\log R)\left(\left(1-\frac{3}{4} \gamma(R)\right)-(1-2 \gamma(R))\right)+O(1) \\
& =\alpha_{R}^{\prime} \log M(a R, f)+(5 / 4) k \alpha(\log R) \gamma(R)+O(1) \\
& \geq \alpha_{R}^{\prime} \log M(a R, f)+O(1) \geq \alpha_{R}^{\prime \prime} \log M(a R, f)
\end{aligned}
$$


In Case II, we note that $t_{1}>R_{0}$ and deduce for large $R$ that

$$
\begin{aligned}
& \log m\left(a t_{1}, f\right)=k \log t_{1}+\log m\left(t_{1}, H\right)+\log |b| \\
& \geq k \log t_{1}+\alpha_{R}^{\prime} \log M(R, F)+\log |b| \\
& \geq k \log t_{1}+\log |b|+\alpha_{R}^{\prime}(\log M(R, H)-\log M(R, G)) \\
& >k \log t_{1}+\log |b|+\alpha_{R}^{\prime}\left(\log M(R, H)-2\left(\frac{1}{R}\right)^{(\alpha-1) / 2}\right) \\
& \geq k \log t_{1}+\alpha_{R}^{\prime}(\log M(a R, f)-k \log R)+O(1) \\
& \geq \alpha_{R}^{\prime} \log M(a R, f)+k\left(\log t_{1}-\alpha_{R}^{\prime} \log R\right)+O(1) \\
& >\alpha_{R}^{\prime} \log M(a R, f)+k\left(\log R_{0}-\alpha_{R}^{\prime} \log R\right)+O(1) \\
& =\alpha_{R}^{\prime} \log M(a R, f)+k \alpha(\log R)((1-\gamma(R))-(1-2 \gamma(R)))+O(1) \\
& =\alpha_{R}^{\prime} \log M(a R, f)+k \alpha \gamma(R) \log R+O(1) \\
& \geq \alpha_{R}^{\prime \prime} \log M(a R, f) .
\end{aligned}
$$

In both cases (5) follows for $f$, with $r=a R_{1}$ or with $r=a t_{1}$ and with $R$ replaced by $a R$.

Note that the required inequality $a R_{1} \leq(a R)^{\alpha}$ is equivalent to $\log R_{1}-\alpha \log R+(\alpha-1)|\log a| \leq 0$, that is,

$$
-(3 / 4) \gamma(R) \alpha(\log R)+(\alpha-1)|\log a| \leq 0,
$$

which holds for all large $R$ since $\gamma(R) \log R \rightarrow \infty$ as $R \rightarrow \infty$ by (4) and the condition (ii) of Theorem 2.1.

For the inequality $a t_{1} \leq(a R)^{\alpha}$ we need to know that we can choose $t_{1} \leq a^{\alpha-1} R^{\alpha}<R^{\alpha}=R_{4}$ since $0<a \leq 1<\alpha$. For this, we need to ensure that (14) holds for all $t \geq a^{\alpha-1} R_{4}$ if $R$ is large enough. If not, then there is $t \in\left[a^{\alpha-1} R_{4}, R_{4}\right)$ for which (14) fails, and then we obtain, as before, since $t^{*} \leq R_{3}$, that

$$
\begin{aligned}
& \log t-\log t^{*} \geq \log \left(a^{\alpha-1} R_{4}\right)-\log R_{3}=(\alpha-1) \log a+\frac{\alpha \gamma(R) \log R}{4} \\
& >\frac{\alpha \gamma(R) \log R}{5} \geq \frac{\alpha \gamma(R)(\log R)(\log t)}{5 \log R_{4}}
\end{aligned}
$$

for all large $R$ since $\gamma(R) \log R \rightarrow \infty$ as $R \rightarrow \infty$. Thus

$$
\begin{aligned}
10 \log N(t, 0, G) & >10 \log N(t, 0, G)-10 \log N\left(t^{*}, 0, G\right) \\
& \geq \frac{\alpha \gamma(R) \log R}{5 \log R_{4}} \log t=\frac{\gamma(R)}{5} \log t,
\end{aligned}
$$

implying that

$$
\gamma(R) \log t<50 \log N(t, 0, G) \leq 50 \log N(t, 0, H)<51 \beta(t) \log t,
$$


which is a contradiction to the condition (ii) of Theorem 2.1 for large $R$ since $t>R$.

This completes the proof of Theorem 2.1.

\section{Proof of Theorem 2.2}

Let the assumptions of Theorem 2.2 be satisfied. Thus we assume that $f$ is a transcendental entire function of order $<1 / 2$. To get a contradiction, we assume that the Fatou set of $f$ contains an unbounded component $D$ that is a simply connected wandering domain. As mentioned in the introduction, all other cases have already been settled.

The proof is divided into two parts. The first part uses dynamics to show that since we are dealing with a component of the Fatou set, the radial spreads of the iterates cannot be too large in a certain sense. The second part uses (6) and shows that the spread must be larger than that after all. These two facts are incompatible, so we get a contradiction, which then completes the proof of Theorem 2.2.

Suppose that $K$ is a compact subset of $D$. In this first part of the proof, our aim is to show that for a certain complex constant $a$ depending only on $D$ and for a possibly large positive number $C>1$ depending on $K$, we have

$$
\frac{1}{C} \leq \frac{\left|f^{j}(z)-a\right|}{\left|f^{j}(w)-a\right|} \leq C
$$

for all $z, w \in K$ and for all $j \geq 0$. This is a consequence of standard estimates for the hyperbolic metric in simply connected domains. This argument is well known (see [1]; [8], Section 10) but we give the details for completeness.

To find $a$, note that since $D$ is a wandering domain, it is disjoint from any of its inverse images. Thus there is a disk $B(a, \tilde{r})=\{z \in \mathbb{C}$ : $|z-a|<\tilde{r}\}$ such that

$$
B(a, \tilde{r}) \cap \cup_{j=0}^{\infty} f^{j}(D)=\emptyset .
$$

Let $D_{j}$ be the component of the Fatou set of $f$ containing $f^{j}(D)$. Note that $D_{j}$ is also an unbounded wandering domain of $f$ and hence is simply connected.

Let $L>1$ be a large constant, to be determined soon. Pick $j \geq 0$ and $z, w \in K$. Suppose that $\left|f^{j}(z)-a\right| /\left|f^{j}(w)-a\right|>L$. Let $\zeta \in \bar{\partial} D_{j}$ be the point closest to $a$, so that in particular, $|\zeta-a|<\left|f^{j}(w)-a\right|$. Let $h_{\Omega}\left(z_{1}, z_{2}\right)$ denote the hyperbolic distance between the points $z_{1}, z_{2}$ of the domain $\Omega$, and let $\lambda_{\Omega}(z)$ denote the density of the hyperbolic 
metric of $\Omega$ at $z \in \Omega$. Thus

$$
h_{D_{j}}\left(f^{j}(z), f^{j}(w)\right) \leq h_{D}(z, w) \leq L_{0}:=\max \left\{h_{D}\left(z_{1}, z_{2}\right): z_{1}, z_{2} \in K\right\} .
$$

Since $D_{j}$ is simply connected, it follows from Koebe's one-quarter theorem that

$$
\lambda_{D_{j}}(z) \geq \frac{1}{4 \operatorname{dist}\left(z, \partial D_{j}\right)} \geq \frac{1}{4|z-\zeta|} \geq \frac{1}{4(|z-a|+|\zeta-a|)}
$$

for all $z \in D_{j}$, where dist $\left(z, \partial D_{j}\right)$ denotes the Euclidean distance of $z$ from $\partial D_{j}$. Hence

$$
\begin{aligned}
L_{0} & \geq h_{D_{j}}\left(f^{j}(z), f^{j}(w)\right) \geq \int_{\left|f^{j}(w)-a\right|}^{\left|f^{j}(z)-a\right|} \frac{d r}{4(r+|\zeta-a|)} \\
& =\frac{1}{4} \log \frac{\left|f^{j}(z)-a\right|+|\zeta-a|}{\left|f^{j}(w)-a\right|+|\zeta-a|} \\
& \geq \frac{1}{4} \log \frac{\left|f^{j}(z)-a\right|+\left|f^{j}(w)-a\right|}{2\left|f^{j}(w)-a\right|} \\
& =\frac{1}{4}\left(\log \left(1+\frac{\left|f^{j}(z)-a\right|}{\left|f^{j}(w)-a\right|}\right)-\log 2\right) \\
& \geq \frac{1}{4}(\log (1+L)-\log 2)
\end{aligned}
$$

which gives a contradiction if $L$ is sufficiently large if compared to $L_{0}$. This proves (15).

Next we observe that even though the constant $C$ depends on $K$ and may be large, we can control the radial spread of the set $f^{j}(K)$ better by using the logarithmic scale.

Suppose that $C_{0}$ is a preassigned constant subject only to $C_{0}>1$. Next we show that by (15) and (16), we have

$$
\frac{1}{C_{0}} \leq \frac{\log \left(2\left|f^{j}(z)-a\right| / \tilde{r}\right)}{\log \left(2\left|f^{j}(w)-a\right| / \tilde{r}\right)} \leq C_{0}
$$

for all $z, w \in K$ and for all sufficiently large $j \geq j_{0}$, say. Having to restrict ourselves to $j \geq j_{0}$ is one cost that we pay in order to get an estimate involving an arbitrary $C_{0}>1$. For if (17) does not hold, then there are sequences $z_{j}, w_{j} \in K$ and integers $n_{j} \rightarrow \infty$ such that

$$
\frac{\log \left(2\left|f^{n_{j}}\left(z_{j}\right)-a\right| / \tilde{r}\right)}{\log \left(2\left|f^{n_{j}}\left(w_{j}\right)-a\right| / \tilde{r}\right)}>C_{0},
$$

that is,

$$
\frac{2\left|f^{n_{j}}\left(z_{j}\right)-a\right|}{\tilde{r}}>\left(\frac{2\left|f^{n_{j}}\left(w_{j}\right)-a\right|}{\tilde{r}}\right)^{C_{0}} .
$$


By passing to a subsequence, we may assume that $\left|f^{n_{j}}\left(z_{j}\right)-a\right| \rightarrow R_{2}$ and $\left|f^{n_{j}}\left(w_{j}\right)-a\right| \rightarrow R_{1}$, say, where $\tilde{r} \leq R_{1}<R_{2}<\infty$ or $R_{1}=R_{2}=\infty$. In the former case, we do not have $f^{n_{j}} \rightarrow \infty$ locally uniformly in $D$, so that by passing to a further subsequence, we may assume that $f^{n_{j}} \rightarrow \omega$ locally uniformly in $D$, where $\omega$ is a complex number with $|\omega-a| \geq \tilde{r}$, by (16). Hence $f^{n_{j}}\left(z_{j}\right) \rightarrow \omega$ and $f^{n_{j}}\left(w_{j}\right) \rightarrow \omega$ as $j \rightarrow \infty$, which contradicts (18). Thus $R_{1}=R_{2}=\infty$. But now, by (15), $\left|f^{n_{j}}\left(z_{j}\right)-a\right| \leq C\left|f^{n_{j}}\left(w_{j}\right)-a\right|<(2 / \tilde{r})^{C_{0}-1}\left|f^{n_{j}}\left(w_{j}\right)-a\right|^{C_{0}}$ when $\left|f^{n_{j}}\left(w_{j}\right)\right|$ is large enough, which is a contradiction. This completes the proof of (17). This also finishes off the first part of the proof of Theorem 2.2. We have now established an upper bound for radial spread, which is effective since the number $C_{0}>1$ is still at our disposal and so we may choose $C_{0}$ to be very close to 1 .

We proceed to show that if we choose $K$ to be of large radial spread, as we may since we are choosing a compact subset of an unbounded domain $D$, then the large radial spread in fact persists under iteration, to the extent that we obtain a contradiction to (17). This contradiction then shows that the domain $D$ with its defining properties could not exist at all, and the proof of Theorem 2.2 is complete.

Suppose that $\alpha, R_{0}$, and $\gamma(r)$ satisfy the conditions of Theorem 2.2. Choose $C_{2}>2$ so large that

$$
\frac{1}{\alpha}<\prod_{n=1}^{\infty}\left(1-\gamma\left(e^{C_{2}^{n}}\right)-C_{2}^{-n} \log ^{+} M(1, f)\right) .
$$

Next pick $R^{\prime}$ so that

$$
r<r^{C_{2}}<M(r, f)
$$

for all $r \geq R^{\prime}$. Choose $R_{1} \geq \max \left\{R_{0}, R^{\prime}\right.$, $\left.\exp \left\{C_{2}\right\}\right\}$ and, in addition, so that $\alpha(1-\gamma(r))>1$ for all $r>R_{1}$. Let $K$ be a compact connected subset of $D$ containing points $z_{0}$ and $w_{0}$ with

$$
\left|w_{0}\right|>\left|z_{0}\right|>R_{1}
$$

and

$$
\frac{\log \left|w_{0}\right|}{\log \left|z_{0}\right|}>\alpha^{2}
$$

Set $K_{n}=f^{n}(K)$. We seek to prove that for each $n \geq 1$, there are points $z_{n}, w_{n} \in K_{n}$ with

$$
\left|w_{n}\right|>\left|z_{n}\right|>R_{1}
$$


and

$$
\frac{\log \left|w_{n}\right|}{\log \left|z_{n}\right|}>\alpha^{2} \prod_{k=1}^{n}\left(1-\gamma\left(e^{C_{2}^{k}}\right)-C_{2}^{-k} \log ^{+} M(1, f)\right)>\alpha .
$$

Since $K$ is connected and (20) holds, there is $\zeta_{0} \in K$ with $\left|w_{0}\right|=$ $\left|\zeta_{0}\right|^{\alpha}$. Thus $\left|\zeta_{0}\right|>\left|z_{0}\right|$. By (6), there is $t \in\left(\left|\zeta_{0}\right|,\left|w_{0}\right|\right]$ with

$$
\frac{\log m(t, f)}{\log M\left(\left|\zeta_{0}\right|, f\right)} \geq \alpha\left(1-\gamma\left(\left|\zeta_{0}\right|\right)\right)
$$

We have

$$
\left|f\left(z_{0}\right)\right| \leq M\left(\left|z_{0}\right|, f\right) .
$$

Take any point $u_{0} \in K$ with $\left|u_{0}\right|=t$. This is possible since $K$ is connected. We have

$$
\frac{\log \left|f\left(u_{0}\right)\right|}{\log \left|f\left(z_{0}\right)\right|} \geq \frac{\log m(t, f)}{\log M\left(\left|z_{0}\right|, f\right)}=\frac{\log m(t, f)}{\log M\left(\left|\zeta_{0}\right|, f\right)} \frac{\log M\left(\left|\zeta_{0}\right|, f\right)}{\log M\left(\left|z_{0}\right|, f\right)}
$$

We next find a lower bound for

$$
\frac{\log M\left(\left|\zeta_{0}\right|, f\right)}{\log M\left(\left|z_{0}\right|, f\right)} \text {. }
$$

We write $\varphi(x)=\log M\left(e^{x}, f\right)$. If $1<r_{1}<r_{2}$ and $x_{j}=\log r_{j}$ for $j=1,2$, and if $r_{1}=\left|z_{0}\right|$ and $r_{2}=\left|\zeta_{0}\right|$, we have

$$
\frac{\log M\left(\left|\zeta_{0}\right|, f\right)}{\log M\left(\left|z_{0}\right|, f\right)}=\frac{\varphi\left(x_{2}\right)}{\varphi\left(x_{1}\right)} .
$$

Since $\varphi$ is convex, we have

$$
\varphi\left(x_{1}\right) \leq \frac{x_{2}-x_{1}}{x_{2}} \varphi(0)+\frac{x_{1}}{x_{2}} \varphi\left(x_{2}\right)
$$

so that

$$
\varphi\left(x_{2}\right) \geq \frac{x_{2}}{x_{1}} \varphi\left(x_{1}\right)-\frac{x_{2}-x_{1}}{x_{1}} \varphi(0)
$$

hence

$$
\frac{\varphi\left(x_{2}\right)}{\varphi\left(x_{1}\right)} \geq \frac{x_{2}}{x_{1}}-\left(\frac{x_{2}}{x_{1}}-1\right) \frac{\varphi(0)}{\varphi\left(x_{1}\right)} .
$$

If $\varphi(0) \leq 0$, we get

$$
\frac{\varphi\left(x_{2}\right)}{\varphi\left(x_{1}\right)} \geq \frac{x_{2}}{x_{1}}
$$

In general, if $\varphi(0)>0$,

$$
\frac{\varphi\left(x_{2}\right)}{\varphi\left(x_{1}\right)} \geq \frac{x_{2}}{x_{1}}\left\{1-\left(1-\frac{x_{1}}{x_{2}}\right) \frac{\varphi(0)}{\varphi\left(x_{1}\right)}\right\} \geq \frac{x_{2}}{x_{1}}\left\{1-\frac{\varphi(0)}{\varphi\left(x_{1}\right)}\right\} .
$$


Thus, whether $\varphi(0) \leq 0$ or $\varphi(0)>0$ we have

$$
\frac{\log M\left(\left|\zeta_{0}\right|, f\right)}{\log M\left(\left|z_{0}\right|, f\right)}=\frac{\varphi\left(x_{2}\right)}{\varphi\left(x_{1}\right)} \geq \frac{\log \left|\zeta_{0}\right|}{\log \left|z_{0}\right|}\left\{1-\frac{\log ^{+} M(1, f)}{\log M\left(\left|z_{0}\right|, f\right)}\right\} .
$$

We conclude that

$$
\begin{aligned}
& \frac{\log \left|f\left(u_{0}\right)\right|}{\log M\left(\left|z_{0}\right|, f\right)} \geq \alpha \frac{\log \left|\zeta_{0}\right|}{\log \left|z_{0}\right|}\left(1-\gamma\left(\left|\zeta_{0}\right|\right)\right)\left(1-\frac{\log ^{+} M(1, f)}{\log M\left(\left|z_{0}\right|, f\right)}\right) \\
= & \frac{\log \left|w_{0}\right|}{\log \left|z_{0}\right|}\left(1-\gamma\left(\left|\zeta_{0}\right|\right)\right)\left(1-\frac{\log ^{+} M(1, f)}{\log M\left(\left|z_{0}\right|, f\right)}\right) \\
\geq & \frac{\log \left|w_{0}\right|}{\log \left|z_{0}\right|}\left(1-\gamma\left(\left|\zeta_{0}\right|\right)-\frac{\log ^{+} M(1, f)}{\log M\left(\left|z_{0}\right|, f\right)}\right) \\
\geq & \frac{\log \left|w_{0}\right|}{\log \left|z_{0}\right|}\left(1-\gamma\left(\left|z_{0}\right|\right)-\frac{\log ^{+} M(1, f)}{\log M\left(\left|z_{0}\right|, f\right)}\right) \\
\geq & \frac{\log \left|w_{0}\right|}{\log \left|z_{0}\right|}\left(1-\gamma\left(\left|z_{0}\right|\right)-C_{2}^{-1} \log ^{+} M(1, f)\right) \\
> & \alpha^{2}\left(1-\gamma\left(e^{C_{2}}\right)-C_{2}^{-1} \log ^{+} M(1, f)\right)>\alpha>1,
\end{aligned}
$$

where in the last inequality we use $\left|z_{0}\right|>\exp \left\{C_{2}\right\}$.

Since $\left|f\left(z_{0}\right)\right| \leq M\left(\left|z_{0}\right|, f\right)$ and $f(K)=K_{1}$ is connected, the set $K_{1}$ contains a point $z_{1}$ with $\left|z_{1}\right|=M\left(\left|z_{0}\right|, f\right)$. We set $f\left(u_{0}\right)=w_{1}$ and note that

$$
\frac{\log \left|w_{1}\right|}{\log \left|z_{1}\right|}>\alpha^{2}\left(1-\gamma\left(e^{C_{2}}\right)-C_{2}^{-1} \log ^{+} M(1, f)\right)>\alpha
$$

To extend this argument from $K_{1}$ to $K_{n}$, we prove the following lemma.

Lemma 4.1. Suppose that $n \geq 1$ and that for all $m$ with $1 \leq m \leq n$, there exist $z_{m}, w_{m} \in K_{m}$ with

$$
\begin{gathered}
\left|w_{m}\right|>\left|z_{m}\right|>R_{1}, \\
\left|z_{m}\right| \geq\left|z_{m-1}\right|^{C_{2}} \geq\left|z_{0}\right|^{C_{2}^{m}}
\end{gathered}
$$

for $1 \leq m \leq n$, and

$$
\kappa_{m} \equiv \frac{\log \left|w_{m}\right|}{\log \left|z_{m}\right|}>\alpha^{2} \prod_{k=1}^{m}\left(1-\gamma\left(e^{C_{2}^{k}}\right)-C_{2}^{-k} \log ^{+} M(1, f)\right)>\alpha .
$$

Then $K_{n+1}$ contains points $z_{n+1}$ and $w_{n+1}$ such that

$$
\begin{gathered}
\left|w_{n+1}\right|>\left|z_{n+1}\right|>R_{1}, \\
\left|z_{n+1}\right| \geq\left|z_{n}\right|^{C_{2}} \geq\left|z_{0}\right|_{2}^{C^{n+1}}
\end{gathered}
$$


and

$$
\kappa_{n+1} \equiv \frac{\log \left|w_{n+1}\right|}{\log \left|z_{n+1}\right|}>\alpha^{2} \prod_{k=1}^{n+1}\left(1-\gamma\left(e^{C_{2}^{k}}\right)-C_{2}^{-k} \log ^{+} M(1, f)\right)>\alpha
$$

Proof of Lemma 4.1. Since $K_{n}$ is connected and

$$
\frac{\log \left|w_{n}\right|}{\log \left|z_{n}\right|}>\alpha
$$

there is $\zeta_{n} \in K_{n}$ with

$$
\frac{\log \left|w_{n}\right|}{\log \left|\zeta_{n}\right|}=\alpha
$$

Now by (6), find $t \in\left(\left|\zeta_{n}\right|,\left|w_{n}\right|\right]=\left(\left|\zeta_{n}\right|,\left|\zeta_{n}\right|^{\alpha}\right]$ with

$$
\frac{\log m(t, f)}{\log M\left(\left|\zeta_{n}\right|, f\right)} \geq \alpha\left(1-\gamma\left(\left|\zeta_{n}\right|\right)\right)>1
$$

Then choose $u_{n} \in K_{n}$ with $\left|u_{n}\right|=t$. Note that $\left|z_{n}\right|<\left|\zeta_{n}\right|$. We have, as before,

$$
\begin{aligned}
& \frac{\log \left|f\left(u_{n}\right)\right|}{\log M\left(\left|z_{n}\right|, f\right)} \geq \frac{\log m(t, f)}{\log M\left(\left|\zeta_{n}\right|, f\right)} \frac{\log M\left(\left|\zeta_{n}\right|, f\right)}{\log M\left(\left|z_{n}\right|, f\right)} \\
& >\alpha\left(1-\gamma\left(\left|\zeta_{n}\right|\right)\right) \frac{\log \left|\zeta_{n}\right|}{\log \left|z_{n}\right|}\left(1-\frac{\log ^{+} M(1, f)}{\log M\left(\left|z_{n}\right|, f\right)}\right) \\
& \geq \frac{\log \left|w_{n}\right|}{\log \left|z_{n}\right|}\left(1-\gamma\left(\left|\zeta_{n}\right|\right)-C_{2}^{-(n+1)} \log ^{+} M(1, f)\right) .
\end{aligned}
$$

Note that

$$
\log \left|\zeta_{n}\right|>\log \left|z_{n}\right| \geq C_{2}^{n} \log \left|z_{0}\right| \geq C_{2}^{n+1} .
$$

Choose $z_{n+1} \in K_{n+1}$ with $\left|z_{n+1}\right|=M\left(\left|z_{n}\right|, f\right)$. This is possible since $K_{n+1}$ is connected, $f\left(z_{n}\right) \in K_{n+1},\left|f\left(z_{n}\right)\right| \leq M\left(\left|z_{n}\right|, f\right)$, and $f\left(u_{n}\right) \in$ $K_{n+1}$ while by $(21)$,

$$
\left|f\left(u_{n}\right)\right| \geq m(t, f) \geq M\left(\left|\zeta_{n}\right|, f\right)>M\left(\left|z_{n}\right|, f\right) .
$$

We get, with $w_{n+1}=f\left(u_{n}\right)$, that

$$
\begin{aligned}
\kappa_{n+1}=\frac{\log \left|w_{n+1}\right|}{\log \left|z_{n+1}\right|} & >\kappa_{n}\left(1-\gamma\left(e^{C_{2}^{n+1}}\right)-C_{2}^{-(n+1)} \log ^{+} M(1, f)\right) \\
& >\alpha^{2} \prod_{k=1}^{n+1}\left(1-\gamma\left(e^{C_{2}^{k}}\right)-C_{2}^{-k} \log ^{+} M(1, f)\right)>\alpha .
\end{aligned}
$$

Also $\left|z_{n+1}\right|=M\left(\left|z_{n}\right|, f\right) \geq\left|z_{n}\right|^{C_{2}}>\left|z_{n}\right|>R_{1}$ by (19). This completes the proof of Lemma 4.1.

We continue with the proof of Theorem 2.2. We have previously shown that the hypothesis of Lemma 4.1 holds for $n=1$. Hence 
induction on $n$ together with Lemma 4.1 shows that for every $n \geq 1$ there are $z_{n}, w_{n} \in K_{n}$ with

$$
\frac{\log \left|w_{n}\right|}{\log \left|z_{n}\right|}>\alpha
$$

The distinction between $\log \left|w_{n}\right|$ and $\log \left(2\left|w_{n}-a\right| / \tilde{r}\right)$ is immaterial and is easily handled by taking $R_{1}$ even larger; we omit the details. Taking $C_{0}$ in (17) with $1<C_{0}<\alpha$ and choosing the appropriate $j_{0}$, we obtain a contradiction as soon as $n \geq j_{0}$. This completes the proof of Theorem 2.2.

\section{Proof of Theorem 2.3}

Let the assumptions of Theorem 2.3 be satisfied. Let $\gamma:(e, \infty) \rightarrow$ $(0, \infty)$ be a decreasing function such that $\gamma(r) / \beta(r) \rightarrow \infty$ as $r \rightarrow \infty$ and

$$
\sum_{n} \gamma\left(e^{2^{n}}\right)<\infty
$$

Since $\gamma(r)$ satisfies the hypothesis of Theorem 2.1, $3 \gamma(r)$ satisfies the hypothesis of Theorem 2.2. Thus Theorem 2.3 now follows from Theorem 2.2 .

\section{Proof of Theorem 2.4}

Let the assumptions of Theorem 2.4 be satisfied. Define

$$
\gamma_{1}(r)=\sup \{\gamma(t): t \geq r\}
$$

for $r>e^{3}$. Then the function $\gamma_{1}$ is decreasing and $\gamma_{1}(r) \geq \gamma(r)$ for all $r>e^{3}$.

We claim that $\beta(r) / \gamma_{1}(r) \rightarrow \infty$ as $r \rightarrow \infty$. Suppose that $r_{n} \rightarrow \infty$. Let $t_{n} \geq r_{n}$ be such that $\gamma\left(t_{n}\right) \geq(1 / 2) \gamma_{1}\left(r_{n}\right)$. Then

$$
\beta\left(r_{n}\right) / \gamma_{1}\left(r_{n}\right) \geq \beta\left(t_{n}\right) / \gamma_{1}\left(r_{n}\right) \geq \beta\left(t_{n}\right) /\left(2 \gamma\left(t_{n}\right)\right),
$$

which tends to infinity by assumption.

For $r>e^{6}$ define $\tilde{\beta}(r)=(1 / 2) \beta(\sqrt{r})$ and $\Gamma(r)=\gamma_{1}(\sqrt{r})$. Note that

$$
\tilde{\beta}(r) \log r=(1 / 2) \beta(\sqrt{r}) \log r=\beta(\sqrt{r}) \log (\sqrt{r})
$$

is a increasing function tending to infinity as $r \rightarrow \infty$. We also have

$$
\tilde{\beta}(r)=(1 / 2) \beta(\sqrt{r})>\frac{1}{2} \frac{1}{(\log \log r-\log 2)^{2}}>\frac{1}{4(\log \log r)^{2}}
$$

for large $r$ by (7).

Let $\tilde{\gamma}(r)$ be a function satisfying $\tilde{\gamma}(r) \geq \Gamma(r), \tilde{\beta}(r) / \tilde{\gamma}(r) \rightarrow \infty$, and $\tilde{\gamma}(r) \log r \rightarrow \infty$ as $r \rightarrow \infty$. 
We choose an increasing sequence $R_{k}$, for $k \geq 1$, satisfying for all $k \geq 2$

$$
\tilde{\gamma}\left(R_{k}\right) \log R_{k}>3 \log R_{k-1}
$$

and

$$
\sum_{j=1}^{k-1} \exp \left\{\frac{\tilde{\beta}\left(R_{j}\right) \log R_{j}}{2}\right\}<\exp \left\{\frac{\tilde{\beta}\left(R_{k}\right) \log R_{k}}{64}\right\} .
$$

By choosing $R_{1}$ sufficiently large, we may and will assume that furthermore,

$$
\begin{aligned}
& 1<\alpha\left(1-3 \tilde{\gamma}\left(R_{k}\right)\right) \quad \text { and } \\
& \alpha\left(1-3 \tilde{\gamma}\left(R_{k+1}\right)\right) \log R_{k+1}>1+\alpha\left(1+\tilde{\gamma}\left(R_{k}\right)\right) \log R_{k}
\end{aligned}
$$

for all $k \geq 1$. We set

$$
\rho_{k}=\frac{\tilde{\beta}\left(R_{k}\right)}{8 \alpha \tilde{\gamma}\left(R_{k}\right)} .
$$

Evidently, $\rho_{k} \rightarrow \infty$ as $k \rightarrow \infty$.

Associate with $R_{k}$ a finite sequence defined as follows. We set

$$
\begin{gathered}
\log r_{0 k}=\alpha\left(1-3 \tilde{\gamma}\left(R_{k}\right)\right) \log R_{k}, \\
\log r_{1 k}=\alpha\left(1-2 \tilde{\gamma}\left(R_{k}\right)\right) \log R_{k}, \\
\log r_{2 k}=\alpha\left(1-\tilde{\gamma}\left(R_{k}\right)\right) \log R_{k}, \\
\log r_{3 k}=\alpha \log R_{k}, \\
\log r_{4 k}=\alpha\left(1+\tilde{\gamma}\left(R_{k}\right)\right) \log R_{k} .
\end{gathered}
$$

Our assumptions (25) guarantee that $R_{k}<r_{0 k}$ and $r_{4 k}<R_{k+1}<$ $r_{0, k+1}$. For large $k$, the ratio $R_{k+1} / r_{4 k}$ is large.

For a real number $x$, we denote by $\lfloor x\rfloor$ the largest integer not exceeding $x$. For $k \geq 1$, let $G_{k}$ be the finite product of Weierstrass factors of genus 0 with positive zeros satisfying

$$
n\left(t, 0, G_{k}\right)=\left\lfloor\left(\frac{t}{r_{0 k}}\right)^{\rho_{k}}\right\rfloor, \quad r_{0 k} \leq t \leq r_{4 k} .
$$

If $m>\rho_{k}+1$, the $m^{\text {th }}$ Fourier coefficient of $\log \left|G_{k}\left(r e^{i \theta}\right)\right|$ is (see [9]), with $n(t)=n\left(t, 0, G_{k}\right)$,

$$
\begin{aligned}
c_{m}\left(r, G_{k}\right) & :=\frac{1}{2 \pi} \int_{0}^{2 \pi} e^{-i m \theta} \log \left|G_{k}\left(r e^{i \theta}\right)\right| d \theta \\
& =\frac{1}{2} \int_{0}^{r}\left(\frac{t}{r}\right)^{m} \frac{n(t)}{t} d t-\frac{1}{2} \int_{r}^{\infty}\left(\frac{r}{t}\right)^{m} \frac{n(t)}{t} d t .
\end{aligned}
$$


We suppose that $r_{0 k}<r \leq r_{3 k}$. Elementary calculations yield

$$
\frac{1}{2} \int_{0}^{r}\left(\frac{t}{r}\right)^{m} \frac{n(t)}{t} d t \leq \frac{1}{2\left(m+\rho_{k}\right)}\left(\frac{r}{r_{0 k}}\right)^{\rho_{k}} .
$$

Since $n(t) \geq\left(t / r_{0 k}\right)^{\rho_{k}}-1$, it is easily seen that

$$
\begin{aligned}
& \frac{1}{2} \int_{r}^{\infty}\left(\frac{r}{t}\right)^{m} \frac{n(t)}{t} d t \geq \frac{1}{2} \int_{r}^{r_{4 k}}\left(\frac{r}{t}\right)^{m} \frac{n(t)}{t} d t \\
\geq & \frac{1}{2\left(m-\rho_{k}\right)}\left(\frac{r}{r_{0 k}}\right)^{\rho_{k}}\left(1-\left(\frac{r}{r_{4 k}}\right)^{m-\rho_{k}}\right)-\frac{1}{2 m} .
\end{aligned}
$$

We now specify a choice of $m$. We choose $m=\left\lfloor\rho_{k}\left(1+\delta_{k}\right)\right\rfloor$ where $2 / \rho_{k}<\delta_{k}<3 / \rho_{k}$. We note that $1<m-\rho_{k}<3$. For this $m$, we have uniformly for $r_{0 k}<r<r_{3 k}$ as $k \rightarrow \infty$ that

$$
\left(\frac{r}{r_{4 k}}\right)^{m-\rho_{k}} \rightarrow 0
$$

and thus for all large $k$ and for all $r \in\left(r_{0 k}, r_{3 k}\right]$,

$$
\frac{1}{2} \int_{r}^{\infty}\left(\frac{r}{t}\right)^{m} \frac{n(t)}{t} d t>\frac{1}{8}\left(\frac{r}{r_{0 k}}\right)^{\rho_{k}}
$$

Combining, we get

$$
\left|c_{m}\left(r, G_{k}\right)\right|>\frac{1}{16}\left(\frac{r}{r_{0 k}}\right)^{\rho_{k}} .
$$

We have the elementary estimate for $r_{0 k}<r \leq r_{3 k}$ that

$$
N\left(r, 0, G_{k}\right) \leq \int_{r_{0 k}}^{r}\left(\frac{t}{r_{0 k}}\right)^{\rho_{k}} \frac{d t}{t}=\frac{1}{\rho_{k}}\left(\left(\frac{r}{r_{0 k}}\right)^{\rho_{k}}-1\right) .
$$

Evidently

$$
N\left(r, 0, G_{k}\right)<8 \delta_{k}\left|c_{m}\left(r, G_{k}\right)\right|
$$

From Jensen's Theorem we now conclude for $r_{0 k}<r \leq r_{3 k}$ and with $m\left(r, 1 / G_{k}\right)$ having its usual Nevanlinna theory meaning as the proximity function of $1 / G_{k}$ that

$$
\begin{aligned}
m\left(r, 1 / G_{k}\right) & =T\left(r, G_{k}\right)-N\left(r, 0, G_{k}\right) \\
& \geq \frac{1}{2}\left|c_{m}\left(r, G_{k}\right)\right|-N\left(r, 0, G_{k}\right) \\
& >\left|c_{m}\left(r, G_{k}\right)\right|\left(\frac{1}{2}-8 \delta_{k}\right) .
\end{aligned}
$$

This implies that

(28) $\log \min \left\{\left|G_{k}\left(r e^{i \theta}\right)\right|: 0 \leq \theta \leq 2 \pi\right\}<-\left|c_{m}\left(r, G_{k}\right)\right|\left(\frac{1}{2}-8 \delta_{k}\right)$. 
We make the following observations:

(i) $n\left(r_{4 k}, 0, G_{k}\right)=\left\lfloor\left(r_{4 k} / r_{0 k}\right)^{\rho_{k}}\right\rfloor$,

or

$$
\begin{aligned}
\log n\left(r_{4 k}, 0, G_{k}\right) & \leq \rho_{k}\left(\log r_{4 k}-\log r_{0 k}\right) \\
& =\rho_{k}\left(4 \alpha \tilde{\gamma}\left(R_{k}\right) \log R_{k}\right)=\frac{\tilde{\beta}\left(R_{k}\right) \log R_{k}}{2} .
\end{aligned}
$$

Thus

$$
n\left(r_{4 k}, 0, G_{k}\right) \leq \exp \left\{\frac{\tilde{\beta}\left(R_{k}\right) \log R_{k}}{2}\right\} .
$$

(ii) For $r_{1 k}<r \leq r_{3 k}$, we have

$$
\begin{aligned}
\log \left|c_{m}\left(r, G_{k}\right)\right| & \geq \rho_{k}\left(\log r-\log r_{0 k}\right)-\log 16 \\
& \geq \frac{\tilde{\beta}\left(R_{k}\right) \log R_{k}}{8}-\log 16>\frac{\tilde{\beta}\left(R_{k}\right) \log R_{k}}{16}
\end{aligned}
$$

by (26) and (27).

For $k \geq 2$, define the polynomial $F_{k}$ by

$$
F_{k}=\prod_{j=1}^{k-1} G_{j}
$$

For $r_{1 k}<r \leq r_{3 k}$ we have

$$
\begin{aligned}
& \log M\left(r, F_{k}\right)=\int_{0}^{r} \log \left(1+\frac{r}{t}\right) d n\left(t, 0, F_{k}\right) \\
& <e \int_{0}^{r}\left(\log \frac{r}{t}\right) d n\left(t, 0, F_{k}\right)=e N\left(r, 0, F_{k}\right) .
\end{aligned}
$$

Thus by (24) and (29)

$$
\begin{aligned}
\log \log M\left(r, F_{k}\right) & <1+\log N\left(r, 0, F_{k}\right) \\
& <1+\log \left(\left(\sum_{j<k} \exp \left\{\frac{\tilde{\beta}\left(R_{j}\right) \log R_{j}}{2}\right\}\right) \log r\right) \\
& <1+\log \left(\exp \left\{\frac{\tilde{\beta}\left(R_{k}\right) \log R_{k}}{64}\right\} \log r\right) \\
& =1+\frac{\tilde{\beta}\left(R_{k}\right) \log R_{k}}{64}+\log \log r \\
& <\frac{\tilde{\beta}\left(R_{k}\right) \log R_{k}}{32},
\end{aligned}
$$

where in the last step we have applied (22). 
We have from (28), (30), and (32) that for large $k$

$$
\begin{aligned}
\log m\left(r, F_{k} G_{k}\right) & \leq \log M\left(r, F_{k}\right)+\log m\left(r, G_{k}\right) \\
& <\exp \left\{\frac{\tilde{\beta}\left(R_{k}\right) \log R_{k}}{32}\right\}-\frac{1}{4} \exp \left\{\frac{\tilde{\beta}\left(R_{k}\right) \log R_{k}}{16}\right\}<0 .
\end{aligned}
$$

We define

$$
f=\prod_{j=1}^{\infty} G_{j}
$$

where the convergence of the infinite product is easily checked and we omit the details; for each $k \geq 2$ we may write

$$
f=F_{k} G_{k} H_{k} \quad \text { where } \quad H_{k}=\prod_{j=k+1}^{\infty} G_{j} .
$$

The above certainly implies that

$$
\log m(r, f)<0, \quad \text { for } \quad r_{1 k}<r \leq r_{3 k} .
$$

Recall that we have required that

$$
\tilde{\gamma}\left(R_{k}\right) \log R_{k}>3 \log R_{k-1} .
$$

For $1 \leq j \leq k-1$ this guarantees that

$$
\tilde{\gamma}\left(R_{k}\right) \log R_{k}>\left(2+\tilde{\gamma}\left(R_{k}\right)\right) \log R_{j},
$$

which is equivalent to

$$
\log R_{k}<\left(1+\frac{1}{2} \tilde{\gamma}\left(R_{k}\right)\right)\left(\log R_{k}-\log R_{j}\right),
$$

or

$$
\frac{\log R_{k}}{\log R_{k}-\log R_{j}}<1+\frac{1}{2} \tilde{\gamma}\left(R_{k}\right)
$$

For $R_{k}<r \leq r_{1 k}$, we have

$$
\begin{aligned}
\log M\left(r, F_{k}\right)-\log M\left(R_{k}, F_{k}\right) & <1+N\left(r, 0, F_{k}\right)-N\left(R_{k}, 0, F_{k}\right) \\
& =1+n\left(R_{k}, 0, F_{k}\right) \log \frac{r}{R_{k}} .
\end{aligned}
$$

Thus

$$
\begin{aligned}
& \frac{\log M\left(r, F_{k}\right)}{\log M\left(R_{k}, F_{k}\right)}-1<\frac{n\left(R_{k}, 0, F_{k}\right)}{\log M\left(R_{k}, F_{k}\right)} \log \frac{r}{R_{k}}+\frac{1}{\log M\left(R_{k}, F_{k}\right)} \\
\leq & \frac{n\left(R_{k}, 0, F_{k}\right)}{\log M\left(R_{k}, F_{k}\right)}\left(\log R_{k}\right)\left(\alpha\left(1-2 \tilde{\gamma}\left(R_{k}\right)\right)-1\right)+\frac{1}{\log M\left(R_{k}, F_{k}\right)} .
\end{aligned}
$$


Now, with $n_{j}$ denoting the total number of zeros of $G_{j}$, we have

$$
\frac{n\left(R_{k}, 0, F_{k}\right)}{\log M\left(R_{k}, F_{k}\right)} \log R_{k} \leq \frac{\sum_{j<k} n_{j} \log R_{k}}{\sum_{j<k} n_{j} \log \left(R_{k} / R_{j}\right)}<1+\frac{1}{2} \tilde{\gamma}\left(R_{k}\right)
$$

by (34). Thus

$$
\begin{aligned}
& \frac{\log M\left(r, F_{k}\right)}{\log M\left(R_{k}, F_{k}\right)}-1 \\
< & \left(1+\frac{1}{2} \tilde{\gamma}\left(R_{k}\right)\right)\left\{\alpha\left(1-2 \tilde{\gamma}\left(R_{k}\right)\right)-1\right\}+\frac{1}{\log M\left(R_{k}, F_{k}\right)} \\
< & \alpha\left(1-\tilde{\gamma}\left(R_{k}\right)\right)-\left(1+\frac{1}{2} \tilde{\gamma}\left(R_{k}\right)\right)+\frac{1}{\log M\left(R_{k}, F_{k}\right)} .
\end{aligned}
$$

Thus for $R_{k}<r \leq r_{1 k}$, we have

$$
\frac{\log M\left(r, F_{k}\right)}{\log M\left(R_{k}, F_{k}\right)}<\alpha\left(1-\tilde{\gamma}\left(R_{k}\right)\right)
$$

where we have used the fact that $\tilde{\gamma}(r) \log r \rightarrow \infty$ as $r \rightarrow \infty$.

For $R_{k}<r \leq r_{1 k}$, we have

$$
\begin{aligned}
& \frac{\log m(r, f)}{\log M\left(R_{k}, f\right)}<\frac{\log m\left(r, F_{k}\right)}{\log M\left(R_{k}, F_{k}\right)}<\frac{\log M\left(r, F_{k}\right)}{\log M\left(R_{k}, F_{k}\right)} \\
& <\alpha\left(1-\tilde{\gamma}\left(R_{k}\right)\right) \leq \alpha\left(1-\Gamma\left(R_{k}\right)\right)=\alpha\left(1-\gamma_{1}\left(\sqrt{R_{k}}\right)\right) \\
& \leq \alpha\left(1-\gamma_{1}\left(R_{k}\right)\right) \leq \alpha\left(1-\gamma\left(R_{k}\right)\right)
\end{aligned}
$$

where we note from $(28)$ that $\log m\left(r, f / F_{k}\right)<0$. The combination of (33) and (35) establishes (9) with $R=R_{k}$.

It remains only to show that $\log \log M(r, f)<\beta(r) \log r$ for all sufficiently large values of $r$. For then, if $t \geq r$, we have

$$
\log \log M(t, f) / \log t<\beta(t) \leq \beta(r)
$$

since the function $\beta(r)$ is decreasing, and (8) follows.

First suppose that $2 r_{4 k}<r<R_{k+1}^{(2 / 3) \alpha}$. We have

$$
\log M\left(r, F_{k} G_{k}\right)<e N\left(r, 0, F_{k} G_{k}\right)
$$

by an argument analogous to that establishing (31). For $j>k$, integration by parts yields

$$
\begin{aligned}
& \log M\left(r, G_{j}\right)=\int_{r_{0 j}}^{r_{4 j}} \log \left(1+\frac{r}{t}\right) d n\left(t, 0, G_{j}\right) \\
& \leq \frac{\rho_{j}}{\rho_{j}-1}\left(\frac{r}{r_{0 j}}\right)\left(\frac{r_{4 j}}{r_{0 j}}\right)^{\rho_{j}-1} .
\end{aligned}
$$


We note that

$$
\log \frac{r}{r_{0 j}} \leq \log \frac{R_{j}^{(2 / 3) \alpha}}{r_{0 j}}<\left(-\frac{1}{8}\right) \log R_{j}
$$

and

$$
\left(\rho_{j}-1\right)\left(\log r_{4 j}-\log r_{0 j}\right)<4 \rho_{j} \alpha \tilde{\gamma}\left(R_{j}\right) \log R_{j}=\frac{1}{2} \tilde{\beta}\left(R_{j}\right) \log R_{j} .
$$

Combining, we conclude that

$$
\log M\left(r, G_{j}\right)<2 \exp \left\{\left(\frac{1}{2} \tilde{\beta}\left(R_{j}\right)-\frac{1}{8}\right)\left(\log R_{j}\right)\right\} .
$$

In view of (23), we deduce for large $r$ that

$$
\log M\left(r, H_{k}\right)=\sum_{j=k+1}^{\infty} \log M\left(r, G_{j}\right)<1 .
$$

From (24) and (29) we have

$$
\begin{aligned}
& \log M(r, f)<e N(r, 0, f)+1<e n(r, 0, f) \log r+1 \\
& <4 \exp \left(\frac{\tilde{\beta}\left(R_{k}\right) \log R_{k}}{2}\right) \log r+1,
\end{aligned}
$$

implying that

$$
\begin{aligned}
& \log \log M(r, f)<\log 8+\frac{\tilde{\beta}\left(R_{k}\right) \log R_{k}}{2}+\log \log r \\
& <\tilde{\beta}(r) \log r=\beta(\sqrt{r}) \log (\sqrt{r}) \leq \beta(r) \log r
\end{aligned}
$$

where we have used (22) and the monotonicity of $\beta(r) \log r$.

Now suppose that $R_{k}^{(2 / 3) \alpha} \leq r \leq 2 r_{4 k}$. It follows from (23) that for all large $k$ we have

$$
2 r_{4 k}<R_{k}^{(4 / 3) \alpha} \leq r^{2} \leq 4 r_{4 k}^{2}<R_{k+1}^{(2 / 3) \alpha} .
$$

Applying an intermediate inequality in (36) to $r^{2}$, we obtain

$$
\log \log M(r, f)<\log \log M\left(r^{2}, f\right)<\beta(r) \log r .
$$

This completes the proof of Theorem 2.4. 


\section{Concluding Remarks}

Theorem 2.4 shows that our minimum modulus approach to the study of the existence of unbounded components of the Fatou set of an entire function cannot be effective for entire functions of order zero whose growth is faster than that specified in Theorem 2.3. A simpler construction shows that this approach cannot be effective for entire functions of order $\rho$ with $0<\rho \leq 1 / 2$. We now outline a construction that shows that no reasonable analogue of Theorem 2.1 exists for entire functions of positive order less than or equal to $1 / 2$. This construction can be modified to produce a function of order $1 / 2$, minimal type.

Theorem 7.1. Suppose that $0<\rho \leq 1 / 2$. There exists an entire function $f$ of order $\rho$ such that if $\alpha>1$, there exist $\alpha^{\prime} \in(0, \alpha)$ and an unbounded sequence $R_{k}$ such that for all $k$

$$
\frac{\log m(r, f)}{\log M\left(R_{k}, f\right)}<\alpha^{\prime}, \quad \text { whenever } \quad R_{k} \leq r \leq R_{k}^{\alpha} .
$$

Proof of Theorem 7.1. We sketch the construction of the required $f$. Let $a_{k}$ be a sequence of real numbers greater than 1 that is dense in $(1, \infty)$. Let $\alpha_{n}$ be a sequence each of whose members is among the $a_{k}$ and such that each $a_{k}$ appears infinitely often in the sequence $\alpha_{n}$.

We choose $\rho^{\prime} \in(0, \rho)$, and set

$$
\beta_{n}=\alpha_{n} \rho^{\prime}, \quad \alpha_{n}^{\prime}=\alpha_{n} \frac{2 \alpha_{n}-2 \beta_{n}}{2 \alpha_{n}-\beta_{n}} .
$$

We note that $\beta_{n}<\alpha_{n}$ and $\alpha_{n}^{\prime}<\alpha_{n}$.

We next choose a rapidly increasing sequence $r_{k}$ with $r_{1}>1$, specifically requiring for $k \geq 2$ that

$$
\log r_{k-1}<\frac{\beta_{k}}{2 \alpha_{k}^{2}} \log r_{k}=\frac{\rho^{\prime}}{2 \alpha_{k}} \log r_{k}
$$

and

$$
\sum_{j=1}^{k-1} r_{j}^{\rho} \log \frac{r_{k}}{r_{j}}<\frac{r_{k}^{\rho-\rho^{\prime}}}{4} .
$$

We set $n_{k}=\left\lfloor r_{k}^{\rho}\right\rfloor$ and define

$$
f(z)=\prod_{k=1}^{\infty}\left(1-\frac{z}{r_{k}}\right)^{n_{k}} .
$$

It is easy to verify that the infinite product converges and defines an entire function of order $\rho$. We note that $f$ has zeros of large multiplicity 
at widely-spaced points on the positive real axis. For each $r>0$ it is evident that $M(r, f)=f(-r)$ and $m(r, f)=f(r)$.

For $k=1,2,3, \ldots$, define

$$
\begin{gathered}
F_{k}(z)=\prod_{j=1}^{k-1}\left(1-\frac{z}{r_{j}}\right)^{n_{j}} \\
G_{k}(z)=\left(1-\frac{z}{r_{k}}\right)^{n_{k}},
\end{gathered}
$$

and

$$
H_{k}(z)=\prod_{j=k+1}^{\infty}\left(1-\frac{z}{r_{j}}\right)^{n_{j}}
$$

For $k \geq 1$, define $R_{k}$ by $R_{k}^{\alpha_{k}}=r_{k}$. Note that $r_{k-1}^{4}<R_{k}$. First suppose that $R_{k} \leq r \leq R_{k}^{\alpha_{k}-\beta_{k}}$. It is elementary that

$$
\begin{aligned}
& \log m(r, f)<\log m\left(r, F_{k}\right)=\sum_{j=1}^{k-1} n_{j} \log \left|1-\frac{r}{r_{j}}\right| \\
& <\left(\alpha_{k}-\beta_{k}\right)\left(\log R_{k}\right) n\left(r_{k-1}, 0, f\right) .
\end{aligned}
$$

Elementary estimates yield

$$
\begin{aligned}
& \log M\left(R_{k}, f\right)>\log M\left(R_{k}, F_{k}\right)=\sum_{j=1}^{k-1} n_{j} \log \left(1+\frac{R_{k}}{r_{j}}\right) \\
& >\sum_{j=1}^{k-1} n_{j}\left(\log R_{k}-\log r_{k-1}\right)>\left(1-\frac{\beta_{k}}{2 \alpha_{k}}\right)\left(\log R_{k}\right) n\left(r_{k-1}, 0, f\right),
\end{aligned}
$$

where we have used (38). We conclude that

$$
\frac{\log m(r, f)}{\log M\left(R_{k}, f\right)}<\frac{\alpha_{k}-\beta_{k}}{1-\frac{\beta_{k}}{2 \alpha_{k}}}=\alpha_{k}^{\prime}, \quad \text { whenever } \quad R_{k} \leq r \leq R_{k}^{\alpha_{k}-\beta_{k}}
$$

Now suppose that $R_{k}^{\alpha_{k}-\beta_{k}}<r \leq R_{k}^{\alpha_{k}}$. We have

$$
\begin{aligned}
& \log m\left(r, G_{k} H_{k}\right)<\log m\left(r, G_{k}\right)=n_{k} \log \left(1-\frac{r}{r_{k}}\right) \\
& <\left(-n_{k}\right) \frac{r}{r_{k}} \leq\left(-n_{k}\right) \frac{R_{k}^{\alpha_{k}-\beta_{k}}}{R_{k}^{\alpha_{k}}}<\left(-\frac{1}{2}\right) \frac{R_{k}^{\alpha_{k} \rho}}{R_{k}^{\beta_{k}}}=\left(-\frac{1}{2}\right) R_{k}^{\alpha_{k}\left(\rho-\rho^{\prime}\right)} .
\end{aligned}
$$


We also have for such $r$ that

$$
\begin{aligned}
& \log M\left(r, F_{k}\right) \leq \log M\left(R_{k}^{\alpha_{k}}, F_{k}\right)=\sum_{j=1}^{k-1} n_{j} \log \left(1+\frac{R_{k}^{\alpha_{k}}}{r_{j}}\right) \\
& <2 \sum_{j=1}^{k-1} n_{j} \log \frac{r_{k}}{r_{j}}<\frac{R_{k}^{\alpha_{k}\left(\rho-\rho^{\prime}\right)}}{2},
\end{aligned}
$$

where we have used (39). We conclude that

(41)

$$
\log m(r, f)<\log M\left(r, F_{k}\right)+\log m\left(r, G_{k} H_{k}\right)<0, R_{k}^{\alpha_{k}-\beta_{k}}<r \leq R_{k}^{\alpha_{k}} .
$$

The combination of (40) and (41) yields

$$
\frac{\log m(r, f)}{\log M\left(R_{k}, f\right)}<\alpha_{k}^{\prime}, \quad \text { whenever } \quad R_{k} \leq r \leq R_{k}^{\alpha_{k}} .
$$

Now suppose that $\alpha>1$ is given. There is a constant subsequence, say $\alpha_{m_{p}}$, of the sequence $\alpha_{k}$ such that for all $p \geq 1$ we have $\alpha_{m_{p}}=$ $\gamma \geq \alpha$. For each $p$, the quantity $\alpha_{m_{p}}^{\prime}$ takes a certain constant value $\gamma^{\prime} \in(0, \gamma)$. Considering how $\gamma^{\prime}$ depends on $\gamma$ via the fixed choice of $\rho^{\prime}$, it is clear that we may choose $\gamma$ so that then $\gamma^{\prime}<\alpha$. Now considering (42) only when $k$ is in the subsequence $m_{p}$, we obtain (37), with the sequence $R_{m_{p}}$ playing the role of the sequence $R_{k}$ required for (37) and with $\gamma^{\prime}$ playing the role of $\alpha^{\prime}$. This completes the proof of Theorem 7.1.

Remark. We see from the proof of Theorem 7.1 that the problematic situation where the minimum modulus is small throughout a long interval occurs immediately before the radii corresponding to the zeros of $f$ of high multiplicity. In the proof of Theorem 2.4 we do not use sparsely occurring multiple zeros, but simple zeros spread in a suitable way within a zone, occurring in sparse zones. This allows us to get a better bound in Theorem 2.4 than would otherwise be possible. For functions of positive order, such refinements are not needed, and the use of multiple zeros is sufficient for our purposes.

\section{REFERENCES}

[1] J.M. Anderson and A. Hinkkanen, Unbounded domains of normality, Proc. Amer. Math. Soc. 126 (1998), 3243-3252.

[2] I.N. Baker, The iteration of polynomials and transcendental entire functions, J. Austral. Math. Soc. (Series A) 30 (1981), 483-495.

[3] I.N. Baker, Wandering domains in the iteration of entire functions, Proc. London Math. Soc. (Series 3) 49 (1984), 563-576.

[4] A.F. Beardon, Iteration of rational functions, Springer-Verlag, Berlin-New York (1991). 
[5] L. Carleson and T. Gamelin, Complex dynamics, Universitext: Tracts in Mathematics, Springer, New York, 1993.

[6] W.K. Hayman, Meromorphic functions, Clarendon Press, Oxford, 1964.

[7] A. Hinkkanen, Entire functions with no unbounded Fatou components, pp. 217-226 in Complex analysis and dynamical systems II, Contemporary Mathematics, vol. 382, AMS, 2005.

[8] A. Hinkkanen, Entire functions with bounded Fatou components, to appear in Transcendental Dynamics and Complex Analysis, Cambridge Univ. Press.

[9] J. Miles and D.F. Shea, An extremal problem in value-distribution theory, Quart. J. Math. (2) 24 (1973), 377-383.

[10] J. Milnor, Dynamics in one complex variable, Introductory lectures, Friedr. Vieweg \& Sohn, Braunschweig, 1999.

[11] P.J. Rippon and G.M. Stallard, Functions of small growth with no unbounded Fatou components, preprint.

[12] G. Stallard, Some problems in the iteration of meromorphic functions, $\mathrm{PhD}$ thesis, Imperial College, London, 1991.

[13] G. Stallard, The iteration of entire functions of small growth, Math. Proc. Cambridge Philos. Soc. 114 (1993), 43-55.

[14] N. Steinmetz, Rational iteration, Complex analytic dynamical systems, de Gruyter Studies in Mathematics, 16, Walter de Gruyter \& Co., Berlin, 1993.

[15] Jian-Hua Zheng, Unbounded domains of normality of entire functions of small growth, Math. Proc. Cambridge Philos. Soc. 128 (2000), 355-361.

University of Illinois at Urbana-Champaign, Department of Mathematics, 1409 West Green Street, Urbana, IL 61801 USA

E-mail address: aimo@uiuc.edu, joe@math.uiuc.edu 